\title{
Alinhamento estático e dinâmico do retropé não diferencia sujeitos com e sem síndrome de dor femoropatelar
}

\author{
Alignment static and dynamic rearfoot does not differentiate subjects \\ with and without patellofemoral pain syndrome
}

\author{
Lisiane Piazza, ${ }^{1 *}$ Aline Crísthna Alves Lisboa, ${ }^{1}$ Thiele de Cássia Libardoni, ${ }^{1}$ Marlon \\ Francys Vidmar, ${ }^{2}$ Luiz Fernando Bortoluzzi de Oliveira, ${ }^{3}$ Gisela Cristina Silva Brinhosa, ${ }^{1}$ \\ Gilmar Moraes Santos ${ }^{1}$
}

ARTIGO ORIGINAL | ORIGINAL ARTICLE

\begin{abstract}
Entre os vários fatores envolvidos na etiologia da Síndrome da Dor Femoropatelar (SDFP) encontramse as alterações no alinhamento do retropé. Este estudo objetivou comparar o alinhamento estático e dinâmico do retropé em sujeitos com e sem SDFP. Participaram 51 sujeitos do sexo feminino, 23 com diagnóstico clínico de SDFP (GSDFP) e 28 clinicamente saudáveis (GC). Os dados foram coletados no Laboratório de Biomecânica da Universidade de Passo Fundo (UPF) - RS e foram avaliados os ângulos do retropé estático de acordo com o protocolo do Software para Avaliação Postural (SAPO), e dinâmico por meio do Software Ariel Performance Analysis System (APAS). Foi utilizada estatística descritiva e inferencial (teste $t$ para amostras independentes e Anova $2 \times 2$ ), com nível de significância de $p \leq 0.05$. Ao comparar o ângulo do retropé estático ( $\mathrm{GSDPF}=7.91^{\circ} \pm 4.45^{\circ} ; \mathrm{GC}=5.92^{\circ} \pm 3.69^{\circ}$ ) e dinâmico $\left(\mathrm{GSDPF}=9.57^{\circ} \pm 3.66^{\circ} ; \mathrm{GC}=9.19^{\circ} \pm 3.08^{\circ}\right)$ entre os grupos, não foram observadas diferenças $(p=$ $0.08)$. Também não foram observadas diferenças entre os grupos $(p=0.22)$ ao comparar-se a variação entre a situação dinâmica e estática $\left(\mathrm{GC}=3.27^{\circ} \pm 4.59^{\circ}\right.$; $\left.\mathrm{GSDFP}=1.65^{\circ} \pm 4.84^{\circ}\right)$ Sujeitos com SDFP não possuem alterações relativas ao alinhamento postural estático e dinâmico do retropé em relação a sujeitos sem esta afecção.

Palavras-chave: síndrome da dor femoropatelar, joelho, retropé
\end{abstract}

ABSTRACT

Among the various factors involved in the etiology of femoropatelar pain syndrome (FPPS) are the changes in hindfoot alignment. This study aimed to compare the static and dynamic alignment of the rearfoot in subjects with and without FPPS. Participants were 51 female subjects, 23 with a clinical diagnosis of FPPS (FPPSG) and 28 clinically healthy (CG). It was evaluated the static angles of the rearfoot in accordance to the protocol for Postural Assessment Software (SAPO), and dynamic with the Ariel Performance Analysis System (APAS). It was computed independent t-test and ANOVA 2-ways $(p \leq 0.05)$. No differences were observed $(p=0.08)$ for the rearfoot angle between between static $\left(\right.$ FPPSG $\left.=7.91^{\circ} \pm 4.45^{\circ} ; \mathrm{CG}=5.92^{\circ} \pm 3.69^{\circ}\right)$ and dynamic $\left(\mathrm{FPPSG}=9.57^{\circ} \pm 3.66^{\circ} ; \mathrm{CG}=9.19^{\circ} \pm 3.08^{\circ}\right.$ ) groups. There were also no differences between groups $(p=0.22)$ comparing the variation between static and dynamic situations $\left(C G=3.27^{\circ} \pm 4.59^{\circ}\right.$; FPPSG $\left.=1.65^{\circ} \pm 4.84^{\circ}\right)$. Subjects with PFPS have no changes in the static and dynamic postural alignment of the rearfoot relative to subjects without this condition.

Keywords: patellofemoral pain syndrome, knee, rearfoot

Artigo recebido a 09.06.2013; $1^{\text {a }}$ Revisão 01.10.2013; 2 ${ }^{\text {a }}$ Revisão 09.01.2014; Aceite 30.01.2014

${ }^{1}$ Universidade do Estado de Santa Catarina (UDESC), Florianópolis, Brasil

${ }^{2}$ Universidade Federal de Ciências da Saúde de Porto Alegre (UFCSPA), Porto Alegre, Brasil

${ }^{3}$ Universidade de Passo Fundo (UPF), Passo Fundo, Brasil

* Autor correspondente: Rua Rio Branco, 1.541, CEP99070-080 Passo Fundo/ Rio Grande do Sul, Brasil; E-mail: lisiane_piazza@yahoo.com.br 


\section{INTRODUÇÃO}

A Síndrome da Dor Femoropatelar (SDFP) caracteriza-se como uma dor difusa na região anterior do joelho, geralmente de início insidioso e progressão lenta, sendo agravada por atividades que aumentam as forças de compressão na articulação femoropatelar, como subir e descer escadas, correr e/ou andar, agachar e ajoelhar, bem como ao permanecer sentado por período prolongado (Thomee, Renstrom, Karlsson, \& Grimby, 1995). Essa condição é geralmente encontrada em atletas e afeta cerca de $20 \%$ da população em geral, sobretudo adolescentes e adultos jovens do sexo feminino (Tumia \& Maffulli, 2002).

Sua etiologia é considerada multifatorial e relacionada a vários fatores que levam ao mau alinhamento patelar (Powers, 2000; Sutvile et al., 2004), além de fraqueza do músculo quadríceps femoral, alterações no alinhamento e na biomecânica dos membros inferiores, especialmente relativas ao ângulo do retropé (Levinger \& Gilleard, 2004; Powers, Maffuci, \& Hampton, 1995; Thijs, Van Tiggelen, Roosen, De Clercq, \& Witvrouw, 2007; Venturini et al., 2006), destacando-se a pronação excessiva da articulação subtalar que leva a compensações biomecânicas com sobrecarga da articulação femoropatelar (Venturini et al., 2006). Segundo Powers (2003) e Vogelbach e Combs (1987), o retropé varo leva à pronação excessiva da subtalar com consequente rotação interna excessiva da tíbia alterando o vetor de força que age sobre a patela gerando maior tensão sobre os tecidos moles laterais e podendo conduzir à dor femoropatelar.

Powers, Maffucci e Hampton (1995), observaram que sujeitos com dor femoropatelar apresentam maior varo do retropé do que sujeitos sem esta afeção. Os autores ressaltam que o varo do retropé pode ser fator contribuinte para a dor femoropatelar, portanto deve ser considerado na avaliação biomecânica da extremidade inferior. No entanto, estes autores avaliaram o ângulo do retropé na posição neutra da subtalar, com o sujeito posicionado em decúbito ventral. Quando a postura do retropé foi avaliada na posição ortostática, Aliberti (2008) e Levinger e Gilleard (2004), encontraram aumento do valgo do retropé em sujeitos com a SDFP, enquanto outros autores não encontraram associação entre a postura do retropé e a ocorrência da SDFP (Messier, Davis, Curl, Lowery, \& Pack, 1991; Thomee et al., 1995).

Como a SDFP é uma afecção que se manifesta principalmente durante atividades dinâmicas (Piazza et al., 2012), torna-se importante conhecer não só as alterações relacionadas ao alinhamento estático dos membros inferiores dos sujeitos com SDFP, mas também as alterações que se manifestam durante suas atividades funcionais dinâmicas.

O conhecimento das diferenças cinemáticas entre indivíduos com e sem SDFP permite desenvolver e aperfeiçoar estratégias de prevenção e tratamento para a SDFP. Barton, Levinger, Menz e Webster (2009) verificaram um atraso no pico de eversão do retropé e adução do antepé, os quais poderiam indicar a existência de uma prolongada pronação em indivíduos com SDFP. Um maior ângulo de eversão do retropé foi encontrado na população com SDFP na passagem do contato do calcanhar, adicionando fortes evidências que diferenças na cinemática do retropé podem estar associadas com a SDFP.

Sabe-se ainda que a avaliação do alinhamento do pé é realizada na prática clínica na avaliação de indivíduos com SDFP, particularmente quando se considera a prescrição de órteses para o pé, no entanto, até a presente data, apenas um estudo (Barton et al., 2011) verificou o alinhamento estático e dinâmico do retropé em indivíduos com SDFP, embora tenha utilizado homens e mulheres na amostra, o que poderia representar viés na análise, uma vez que existem diferenças biomecânicas entre os sexos (Csintalan, Schulz, Woo, Macmahon, \& Lee, 2002). Assim, surge a necessidade de novos estudos que avaliem não só o alinhamento estático de sujeitos com SDFP, mas também o alinhamento dinâmico do retropé, proporcionando desta forma, maior conheci- 
mento para o processo de avaliação e reabilitação desses sujeitos.

Face ao exposto, este estudo teve como objetivo comparar o alinhamento postural estático e dinâmico do retropé de mulheres com e sem Síndrome da Dor Femoropatelar. Justificase a amostra somente do sexo feminino para se evitar um possível viés nos resultados, uma vez que existem diferenças biomecânicas entre os sexos (Csintalan et al., 2002; Powers, 2000) e também pela maior incidência da SDFP neste sexo (Tumia \& Maffulli, 2002).

\section{MÉTODO}

Este estudo foi aprovado pelo Comitê de Ética e Pesquisa da Universidade do Estado de Santa Catarina sob protocolo 33/2010. Os dados foram coletados no Laboratório de Biomecânica da Universidade de Passo Fundo (UPF) - RS, no período de janeiro a maio de 2011.

\section{Participantes}

Foram escolhidos de forma não probabilística intencional 55 sujeitos do sexo feminino
(Marconi \& Lakatos, 2009). Destes, foram excluídos quatro por não se enquadrarem nos critérios de inclusão do estudo. Após, os 51 sujeitos foram divididos em dois grupos, 23 sujeitos com SDFP (GSDFP) e 28 clinicamente saudáveis (GC). As características dos sujeitos nos dois grupos são mostradas na Tabela 1 .

Os critérios de inclusão para o grupo com SDFP (GSDFP) foram apresentar dor anterior ou retropatelar, exacerbada por pelo menos três das seguintes situações: subir ou descer escadas, agachar por tempo prolongado, ajoelhar, correr, permanecer sentado por longos períodos, ao contrair de forma estática o quadríceps femoral e ao praticar desporto (Cowan, Bennell, \& Hodges, 2002; Loudon, Wiesner, Goist-Foley, Aajes \& Loudon, 2002); início insidioso dos sintomas sem relação com um evento traumático (Cabral, Melim, Sacco, \& Marques, 2008); dor igual ou maior que $2 \mathrm{~cm}$ na Escala Visual Analógica (EVA: 0-10 cm) na articulação femoropatelar nos sete dias que precederam o teste, durante a realização das atividades supracitadas (Santos, 2006); dor, de qualquer grandeza, em dois testes funcionais

Tabela 1

Características dos participantes

\begin{tabular}{ccc}
\hline Características & GSDFP $(\mathrm{n}=23)$ & GC $(\mathrm{n}=28)$ \\
\hline Idade (anos) ${ }^{*}$ & $22.04 \pm 5.66$ & $21.46 \pm 3.79$ \\
Massa Corporal $(\mathrm{kg}){ }^{*}$ & $58.95 \pm 8.13$ & $59.53 \pm 8.28$ \\
Estatura $(\mathrm{m}){ }^{*}$ & $1.65 \pm 0.07$ & $1.64 \pm 0.05$ \\
Dominância & Membro inferior direito: 22 (95.4\%) & Membro inferior direito: $26(92.8 \%)$ \\
& Membro inferior esquerdo: $1(4.6 \%)$ & Membro inferior esquerdo: $2(7.2 \%)$ \\
Membro com dor & Unilateral (joelho direito): 4 (17.4\%) & - \\
& Unilateral (joelho esquerdo): 4 (17.4\%) & - \\
& Bilateral (+ intensa joelho direito): & - \\
& 13 (56.5\%) & - \\
Início dos & Bilateral (+ intensa joelho esquerdo): & - \\
sintomas & Menos de 2 anos: 11 (47.8\%) & - \\
& 2 a 4 anos: 8 (34.8\%) & - \\
\hline
\end{tabular}

Nota: ${ }^{*} p>0.05$ segundo o teste $t$ para amostras independentes 
com duração de 30 segundos cada um (agachar a 90 graus e descer um step com $25 \mathrm{~cm}$ de altura) (Cowan et al., 2002). Além de se enquadrar nos critérios de inclusão, os sujeitos do GSDFP possuíam diagnóstico clínico de Síndrome da Dor Femoropatelar feito por um médico ortopedista, especialista em joelho.

Os critérios de inclusão para o grupo controle (GC) foram: ausência de história de lesão meniscal ou ligamentar, trauma, cirurgia ou fratura do membro inferior (Loudon et al., 2002; Powers, 2000); sem história de dor na articulação do joelho ou na articulação femoropatelar (dor $0 \mathrm{~cm}$ na EVA) (Powers, 2000); ausência de qualquer problema nas articulações na bacia e pé, doença neurológica ou do sistema osteomioarticular (Loudon et al., 2002); não ter realizado tratamento fisioterapêutico no membro inferior; sem dor, de qualquer grandeza, durante a realização dos testes funcionais com duração de 30 segundos cada um (agachar a 90 graus; descer um step com $25 \mathrm{~cm}$ de altura) (Cowan et al., 2002).

Os critérios de exclusão para ambos os grupos foram: presença de doença neurológica (Laprade, Culham, \& Brouwer, 1998); história de trauma nos membros inferiores, lesão meniscal ou ligamentar do joelho (Cowan et al., 2002); luxação patelar recidivante; história de cirurgia no joelho ou membros inferiores (Powers, 2000); presença de doenças sistémicas que pudessem comprometer a locomoção (Aliberti, 2008).

\section{Instrumentos}

A análise do ângulo do retropé estático foi realizada através do Software para Avaliação Postural (SAPO - versão 6.8), sendo utilizada para o registro das imagens uma câmara fotográfica (Sony Cyber Shot 14.1 MP) e a calibração das imagens foi realizada através de um fio de prumo preso no teto, sobre o qual foram colocadas duas bolas de isopor com uma distância de $1 \mathrm{~m}$ entre elas.

A análise do ângulo do retropé dinâmico foi realizada pelo Software Ariel Performance Analysis System (APAS), sendo as imagens adquiridas através de uma câmara de filmar digital (Sony HandyCam DCR-SR65), com frequência de aquisição de $60 \mathrm{~Hz}$. Para esta avaliação utilizou-se um sistema de calibração bidimensional de oito pontos, de dimensões de $61 \mathrm{~cm}$ no eixo $x$ e $80 \mathrm{~cm}$ no eixo $y$ e um ponto fixo foi posicionado ao lado do calibrador.

Em ambas avaliações (estática e dinâmica) foram utilizados marcadores esféricos no membro inferior dos sujeitos para avaliação dos ângulos.

\section{Procedimentos}

Inicialmente, os sujeitos foram esclarecidos quanto à importância da pesquisa, critérios de inclusão e exclusão, bem como sua participação nesta através do Termo de Consentimento Livre e Esclarecido e Termo de Consentimento para Fotografias, Vídeos e Gravações. Após, foi aplicada uma ficha de avaliação para caracterização dos sujeitos e realizada a avaliação do ângulo do retropé estático, por meio do protocolo do SAPO (versão 0.68), onde foram demarcados, com pequenas bolas de isopor e fita dupla face, o ponto médio do terço póstero-inferior da perna, ponto médio do tendão de Aquiles na altura dos dois maléolos e ponto médio do calcâneo (SAPO, 2010).

Uma máquina fotográfica (Sony Cyber Shot $14.1 \mathrm{Mp}$ ) foi posicionada em um tripé com altura de $95 \mathrm{~cm}$ e como referência vertical foi fixado um fio-de-prumo no teto. Sobre esse fio foram colocadas duas bolas de isopor com uma distância de $1 \mathrm{~m}$ entre elas, que serviram como sistema de calibração. O sujeito e o fio-deprumo foram posicionados num mesmo plano perpendicular ao eixo da câmara a uma distância de 3 metros dessa (SAPO, 2010). Os pés dos sujeitos foram dispostos de forma paralela e em posição neutra, distantes $10 \mathrm{~cm}$ um do outro, sendo esta distância mensurada com uma régua e demarcada no solo. Em seguida, foram adquiridas imagens digitais no plano frontal, vista posterior. Dando continuidade, foi realizada a avaliação do ângulo do retropé dinâmico durante a marcha em superfície plana. Foi utilizada uma câmara de filmar digital 
(Sony HandyCam DCR-SR65), posicionada num tripé a uma altura de $50 \mathrm{~cm}$ do solo e distância de $3 \mathrm{~m}$ do sujeito, sendo as imagens adquiridas no plano frontal, vista posterior do sujeito.

Quatro marcadores esféricos foram colocados no sujeito, nos seguintes pontos anatómicos: um marcador no centro do calcanhar, logo acima da sola da sapatilha (1), outro no centro do calcanhar, na inserção do tendão de Aquiles (2), um terceiro no centro do tendão de Aquiles na altura do maléolo medial (3) e um quarto $15 \mathrm{~cm}$ acima do terceiro marcador no centro da perna (4) (Cheung \& Ng, 2007; De Wit, De Clercq, \& Aerts, 2000; Perry \& Lafortune, 1995; Santos, 2008) (Figura 1).

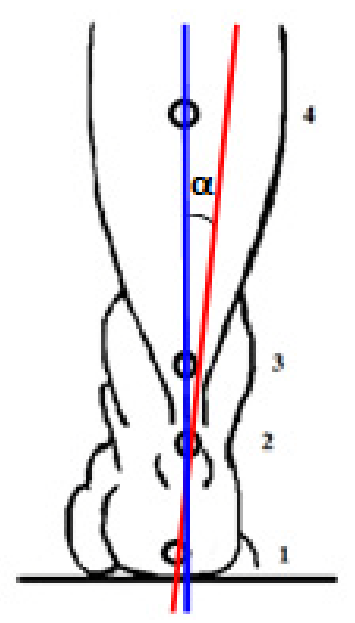

Figura 1. Posicionamento dos marcadores para o cálculo do ângulo do retropé: 1 - logo acima da sola da sapatilha; 2 - centro do calcanhar, na inserção do tendão de Aquiles; 3- centro do tendão de Aquiles na altura do maléolo medial; $4-15 \mathrm{~cm}$ acima do terceiro marcador no centro da perna

Para realizar as marcações para colocação dos marcadores foi solicitado ao sujeito para permanecer em pé, em posição ortostática, com o peso distribuído de forma igual em ambos os pés. Para garantir o mesmo alinhamento de todos os sujeitos durante as marcações, estes foram instruídos a posicionar o pé sobre uma linha reta marcada no solo, fazendo com que o centro do calcanhar ficasse no meio dessa linha e também o segundo dedo posicionado sobre a mesma (Santos, 2008). No
GSDFP com dor bilateral, foi avaliado o membro com maior exacerbação da dor no momento da avaliação, nos sujeitos com dor unilateral foi considerado apenas aquele membro. No GC foi avaliado o membro dominante. O sujeito foi orientado a caminhar ao longo de uma distância de 8 metros, de forma natural. Foram gravados cinco ensaios consecutivos da marcha a uma velocidade homogénea. Para o cálculo das coordenadas reais, foi colocado um sistema de calibração bidimensional no plano das filmagens, constituído por oito pontos distando entre si $61 \mathrm{~cm}$ no eixo horizontal e $80 \mathrm{~cm}$ no eixo vertical e um ponto fixo foi colocado ao lado do calibrador.

\section{Análise dos Dados}

Para análise do ângulo do retropé estático foram realizadas três medidas e feita uma média entre estas três, sendo as imagens calibradas e digitalizadas de acordo com o protocolo do SAPO e posteriormente os dados foram armazenados no próprio software.

Para a análise cinemática do retropé, a digitalização das imagens foi realizada no Software APAS e os dados filtrados digitalmente com uma frequência de corte de $6 \mathrm{~Hz}$. Foi analisado o valor máximo do ângulo do retropé durante a fase de apoio simples da marcha no intervalo de $30 \%$ a $50 \%$ da fase de apoio da marcha (Perry, 2005), sendo o ângulo do retropé formado pela intersecção das linhas que formam o segmento perna com o segmento pé (Figura 1), sendo a eversão considerada positiva e a inversão negativa. A fase de apoio da marcha foi considerada do instante do toque do calcanhar ao solo até o desprendimento dos dedos (Whittle, 1993).

Um único passo foi considerado em cada ensaio, totalizando 5 passos ao final. A escolha por esse número de passos baseou-se em estudos prévios da análise cinemática do retropé. (Chuter, 2010; Cornwall \& Mcpoil, 1995; Moseley, Smith, Hunt, \& Grant, 1996) e no estudo de Diss (2001) que observou uma fiabilidade acima de 0.93 para as variáveis cinemáticas numa análise de 5 passos. 
Os dados cinemáticos foram normalizados na base do tempo, ajustada de $0 \%$ a $100 \%$ para a fase de apoio da marcha, com intervalos de $1 \%$ usando como referência o instante do toque do calcanhar até o desprendimento dos dedos do solo. Para esta normalização foi utilizada uma rotina elaborada no programa Matlab.

Em ambas avaliações (estática e dinâmica) foram analisados os ângulos relativos entre os segmentos perna e pé.

\section{Análise Estatística}

Para a análise estatística foi utilizado o Statistical Package for the Social Sciences (SPSS v. 17.0). A estatística descritiva foi utilizada para caracterização dos sujeitos. O teste de ShapiroWilk mostrou que os dados apresentavam distribuição gaussiana $(p=0.96)$; o teste $t$ de Student para amostras independentes foi utilizado para testar a homogeneidade dos sujeitos em relação à idade, massa, estatura e velocidade da marcha, bem como comparar a variação entre as condições estática e dinâmica do ângulo do retropé entre o GSDFP e GC e a Anova $2 \times 2$ para comparar o ângulo do retropé estático e dinâmico entre o GSDFP e GC. O nível de significância adotado foi de $p \leq 0.05$. Para calcular o effect size, foi utilizado o programa Gpower 3.1, usando-se os valores de média e desvio padrão dos grupos.

\section{RESULTADOS}

Ao comparar o ângulo do retropé estático e dinâmico entre o GSDFP e o GC, conforme se pode observar na tabela 2, não foram observadas diferenças entre os grupos em relação aos ângulos do retropé estático e dinâmico.
A figura 2 apresenta a variação do ângulo do retropé entre as situações estática e dinâmica. Constatou-se uma maior variação entre as duas situações no GC $\left(3.27 \pm 4.59^{\circ}\right)$ em relação ao GSDFP $\left(1.65 \pm 4.84^{\circ}\right)$, no entanto ao comparar esta variação entre os grupos, não foi observada diferença significativa $(p=0.22)$.

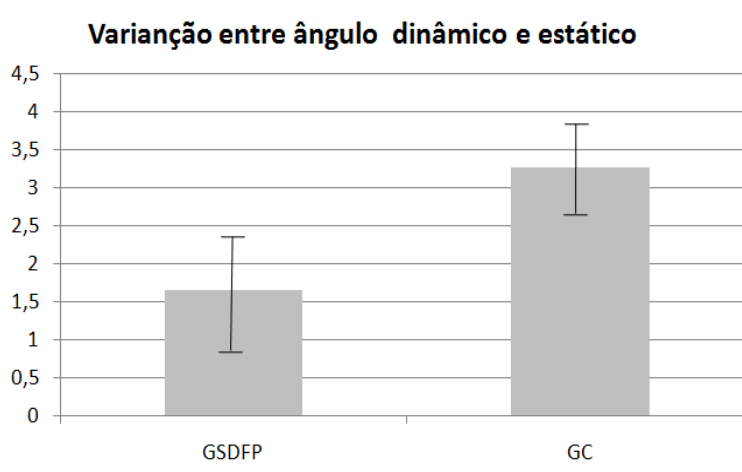

Figura 2. Média \pm desvio padrão da variação do ângulo máximo de eversão do retropé (graus) entre as situações dinâmica e estática do GSDFP e GC

\section{DISCUSSÃO}

Este estudo teve como objetivo comparar o alinhamento postural estático e dinâmico do retropé nos sujeitos com e sem SDFP. Ao comparar o ângulo do retropé estático e o ângulo do retropé dinâmico entre o GSDFP e o GC, não foram observadas diferenças, o que sugere cautela na utilização deste ângulo como fator para o desenvolvimento ou agravo da SDFP. Estes resultados vão ao encontro de Aliberti (2008), que ao avaliar o ângulo do retropé estático através do SAPO, também não observou alterações neste ângulo nos sujeitos com SDFP. O autor explica estes resultados pelo fato das medidas terem sido realizadas de forma estática e os sinais e sintomas da SDFP

Tabela 2

Comparação do ângulo do retropé estático e dinâmico (graus) entre os sujeitos do grupo controle e com SDFP

\begin{tabular}{|c|c|c|c|c|c|}
\hline & $\begin{array}{l}\text { Grupo com Síndrome } \\
\text { da Dor Femoropatelar } \\
\qquad \mathrm{M} \pm \mathrm{DP}\end{array}$ & $\begin{array}{l}\text { Grupo Controle } \\
\qquad \mathrm{M} \pm \mathrm{DP}\end{array}$ & $F$ & $p$ & Effect Size \\
\hline Ângulo retropé estático & $7.91 \pm 4.45^{\circ}$ & $5.92 \pm 3.69^{\circ}$ & \multirow{2}{*}{3.06} & \multirow{2}{*}{0.08} & 0.06 \\
\hline Ângulo retropé dinâmico & $9.57 \pm 3.66^{\circ}$ & $9.19 \pm 3.08^{\circ}$ & & & 0.23 \\
\hline
\end{tabular}


exacerbarem-se principalmente durante atividades dinâmicas.

Entretanto, Belchior, Arakaki, Reis e Carvalho (2006), Venturini, Morato, Michetti, Russo e Carvalho (2006) e Powers et al. (1995) encontraram alterações no ângulo do retropé estático nos sujeitos com e sem SDFP, sugerindo que alterações nestes ângulos poderiam contribuir para o desenvolvimento ou agravo da SDFP. Sendo assim, existem resultados distintos na literatura a respeito da postura estática do pé em sujeitos com SDFP e sua contribuição para esta afeção (Messier et al., 1991; Powers et al.,1995; Sutlive et al., 2004; Thomee et al., 1995; Witvrouw, Lysens, Bellemans, Cambier, \& Vanderstraeten, 2000).

É possível que a natureza multifatorial da síndrome possa contribuir para os resultados controversos entre os diferentes estudos, sugerindo a existência de subgrupos que por sua vez poderiam apresentar características diferentes entre si, como já ocorre em pacientes submetidos à reconstrução do ligamento cruzado anterior (Alkjaer et al., 2002; Chmielewski, Hurd, \& Snyder-Mackler, 2005). Adicionalmente, a SDFP é uma afeção que se manifesta principalmente durante atividades dinâmicas, sendo fundamental a avaliação cinemática entre sujeitos com e sem a SDFP durante estas atividades (Barton et al., 2009).

No presente estudo, também não foram observadas diferenças no ângulo do retropé dinâmico entre os sujeitos com e sem SDFP. Assim, a SDFP parece também não estar relacionada com alterações na magnitude das variáveis cinemáticas deste ângulo. Esses resultados vão ao encontro daqueles encontrados por Levinger e Gilleard (2007), os quais também não observaram diferenças na magnitude da eversão do retropé ao realizar uma análise do movimento tridimensional da tíbia e do retropé em sujeitos com e sem SDFP.

Ao comparar a variação entre as situações dinâmica e estática entre os grupos, o GC apresentou maior variação entre as condições $\left(3.27^{\circ}\right)$ do que o GSDPF $\left(1.65^{\circ}\right)$, embora esta diferença não tenha sido significativa. É possí- vel que a menor amplitude de movimento do retropé, entre as condições estática e dinâmica, no GSDFP, tenha ocorrido pela presença de dor, que limitaria os movimentos desta articulação nos sujeitos com SDFP, fator não presente nos sujeitos do GC.

Além disso, Tiberio (1987) coloca que como o ciclo da marcha não ocorre somente em sequência, mas necessita de um sincronismo entre as articulações, um movimento anormal da subtalar modificará a biomecânica do joelho. Assim, uma pronação excessiva poderá atrasar a rotação externa da perna que acompanha a supinação da subtalar, onde este atraso resulta em uma reação compensatória na articulação tibiofemoral levando a presença de sintomas femoropatelares. Segundo Powers et al. (1995) e Grenholm, Stensdotter e HagerRoss (2009), várias atividades funcionais praticadas pelos sujeitos com SDFP poderiam resultar em modificações nos padrões de caminhar, devido à sensação de dor ou desconforto, sendo estas estratégias para reduzir as demandas musculares e os sintomas da dor. Assim, acreditamos que esta menor variação entre o ângulo estático e dinâmico também poderia ser uma estratégia adotada pelos sujeitos com SDPF em função da sua dor.

No presente estudo optamos por realizar as avaliações com os sujeitos utilizando uma sapatilha padrão com o objetivo de aproximálos o máximo possível da realidade, tendo em vista que a maioria das atividades realizadas no dia-a-dia é com calçado. No entanto, acreditamos que o uso da sapatilha possa ter influenciado nos resultados, o que pode ser considerada uma limitação do estudo. Também optámos por utilizar dois softwares para avaliação dos ângulos (SAPO e APAS) devido à logística das avaliações, o que também pode ser considerada uma limitação. O fato da avaliação cinemática ser realizada somente no plano frontal, além da identificação imprecisa do início e final do passo que são as limitações dos instrumentos, também são consideradas limitações do presente estudo. 
O presente estudo contribui para elucidar a questão relativa ao alinhamento estático e dinâmico do retropé na SDFP, uma vez que estas medidas são amplamente realizadas na prática clínica em sujeitos com essa afeção. Através deste estudo observou-se que sujeitos com SDFP não possuem diferenças na magnitude destes ângulos em relação a sujeitos assintomáticos, sugerindo que a SDFP não possui relação com alterações no alinhamento do retropé.

\section{CONCLUSÕES}

Os resultados do presente estudo, nas condições experimentais utilizadas, evidenciaram não haver alterações no alinhamento postural estático e dinâmico do retropé nos sujeitos com Síndrome da Dor Femoropatelar em relação ao grupo assintomático.

\section{Agradecimentos:}

Nada a declarar.

\section{Conflito de Interesses:}

Nada a declarar.

Financiamento:

Nada a declarar.

\section{REFERÊNCIAS}

Aliberti, S. (2008). Influência da síndrome da dor patelofemural no alinhamento postural dos membros inferiores e na distribuição da pressão plantar durante a marcha e descer escadas. Dissertação Mestrado, Faculdade de Medicina, Universidade de São Paulo, Brasil.

Alkjaer, T., Simonse, E. B., Peter Magnusson, S.P., Agaard, H., \& Dyrhre-Poulen, P. (2002). Differences in the movement pattern of a forward lunge in two types of anterior cruciate ligament deficient patients: Copers and non-copers. Clinical Biomechanics, 17(8), 586-593.

Barton, C. J., Levinger, P., Menz, H. B., \& Webster, K. E. (2009). Kinematic gait characteristics associated with patellofemoral pain syndrome: A systematic review. Gait $\mathcal{E}$ Posture, 30, 405-416.
Barton, C.J., Levinger, P., Crossley, K.M., Webster, K.E., \& Menz, H.B. (2011). Relationships between the foot posture index and foot kinematics during gait in individuals with and without patellofemoral pain syndrome. Journal of Foot and Ankle Research, 4(10), 1-7. doi: 10.1186/ 1757-1146-4-10

Belchior, A.C.G., Arakaki, J.C., Reis, F.A., \& Carvalho, P.T.C. (2006). Efeitos na medida do ângulo Q com a contração isométrica voluntária máxima do músculo quadricipital. Revista Brasileira de Medicina do Esporte, 12(1), 6-10. doi: 10.1590/S1517-86922006000100002

Cabral, C.M., Melim, Â.M., Sacco, I.N., \& Marques, A.P. (2008). Fisioterapia em pacientes com síndrome fêmoro-patelar: Comparação de exercícios em cadeia cinética aberta e fechada. Acta Ortopédica Brasileira, 16(3), 180-185. doi: 10.1590/s1413-78522008000300012

Cheung, R.T., \& Ng, G.Y. (2007). Efficacy of motion control shoes for reducing excessive rearfoot motion in fatigued runners. Physical Therapy in Sport, 8, 75-81. doi: 10.1016/2006.12.002

Chmielewski, T.L., Hurd, W.J., \& Snyder-Mackler, L. (2005). Elucidation of a potentially destabilizing control strategy in ACL deficient noncopers. Journal of Electromyography Kinesiology, 15(1), 83-92. doi: 10.1016/s1050-6411(04)00 073-2

Chuter, V.H. (2010). Relationships between foot type and dynamic rearfoot frontal plane motion. Journal of Foot and Ankle Research, 3(9), 1-6. doi: 10.1186/1757-1146-3-9

Cornwall, M.W. \& Mcpoil, T. (1995). Comparison of 2-dimensional and 3-dimensional rear foot motion during walking. Clinical Biomechanics, 10(1), 36-40. doi: 10.1016/0268-0033(95)904 $35-\mathrm{C}$

Cowan, S.M., Bennell, K.L., \& Hodges, P.W. (2002). Therapeutic patellar taping changes the timing of vasti muscle activation in people with patellofemoral pain syndrome. Clinical Journal of Sport Medicine, 12, 339-347.

Csintalan, R.R., Schulz, M.M., Woo, J., Macmahon, P.J. \& Lee, T.Q. (2002). Gender differences in patellofemoral joint biomechanics. Clinical Orthopaedics and Related Research, 402, 260-269.

De Wit, B., De Clercq, D., \& Aerts, P. (2000). Biomechanical analysis of the stance phase during barefoot and shod running. Journal of Biomechanics, 33, 269-278. doi: 10.1016/s0021-9290(99) 00192-x 
Diss, C.E. (2011). The reliability of kinetic and kinematic variables used to analyse normal running gait. Gait \& Posture, 14, 98-103.

Grenholm, A., Stensdotter, A,. \& Hager-Ross, C. (2009). Kinematic analyses during stair descent in young women with patellofemoral pain. Clinical Biomechanics, 24, 88-94. doi:10.1016/2008.09.004.

Laprade, J., Culham, E., \& Brouwer, B. (1998). Comparison of five isometric exercises in the recruitment of the vastus medialis oblique in persons with and without patellofemoral pain syndrome. Journal of Orthopaedic \& Sports Physical Therapy, 27(3), 197-204.

Levinger, P., \& Gilleard, W. (2004). An evaluation of the rearfoot posture in individuals with patellofemoral pain syndrome. Journal of Sports Science and Medicine, 3(1), 8-14.

Levinger, P., \& Gilleard, W. (2007). Tibia and rearfoot motion and ground reaction forces in subjects with patellofemoral pain syndrome during walking. Gait \& Posture, 24(1), 2-8. doi:10.1016/2005.12.015.

Loudon, J.K., Wiesner, D., Goist-Foley, H.L., Aajes, C., \& Loudon, K.L. (2002). Intra-rater reliability of functional performance tests for subjects with patellofemoral pain syndrome. Journal of Athletic Training, 37(3), 256-261.

Marconi, M.A., \& Lakatos, E.M. (2009). Técnicas de Pesquisa (7a ed.). São Paulo: Atlas.

Messier, S.P., Davis, S.E., Curl, W.W., Lowery, R.B., \& Pack, R.I. (1991). Etiologic factors associated with patellofemoral pain in runners. Medicine $\mathcal{E}$ Science in Sports \& Exercise, 23, 1008-1015.

Moseley, L., Smith, R., Hunt, A., \& Grant, R. (1996). Three-dimensional kinematics of the rearfoot during the stance phase of walking in normal young adult males. Clinical Biomechanics, 11(1), 39-45. doi:10.1016/0268-0033(95)000 36-4

Perry, J. (2005). Análise da marcha: Sistemas de Análise da Marcha (v3). Barueri: Manole.

Perry, S.D., \& Lafortune, M.A. (1995). Influences of inversion/eversion of the foot upon impact loading during locomotion. Clinical Biomechanics, 10(5), 253-257. doi:10.1016/0268-0033(95)0 0006-7

Piazza, L., Lisboa, A.C.A., Costa, V., Brinhosa, G.C., Vidmar, M.F., Oliveira, L.F.B., Libardoni, T.C., \& Santos, G.M. (2012). Sintomas e limitações funcionais de pacientes com síndrome da dor patelofemoral. Revista Dor, 13(1), 50-52.
Powers, C.M. (2000). Patellar Kinematics, Part I: The influence of vastus muscle activity in subjects with and without patellofemoral pain. Physical Therapy, 80(10), 956-964.

Powers, C.M. (2003). The influence of altered lower-extremity kinematics on patellofemoral joint dysfunction: A theoretical perspective. Journal of Orthopaedic \& Sports Physical Therapy, 33(11), 639-646.

Powers, C.M., Maffucci, R., \& Hampton, S. (1995). Rearfoot posture in subjects with patellofemoral pain. Journal of Orthopaedic \& Sports Physical Therapy, 22(4), 155-160.

Santos, G.M. (2006). Controle motor do joelho durante caminhar na esteira em sujeitos com e sem dor femoropatelar. Tese de Doutorado da Universidade Federal de São Carlos, Brasil.

Santos, J.O.L. (2008). Aspectos cinemáticos e cinéticos do movimento de eversão do calcanhar durante a marcha. Dissertação de Mestrado, Universidade do Estado de Santa Catarina, Brasil.

SAPO (2010). Portal do Software para Avaliação Postural. São Paulo: Incubadora Virtual FAPESP.

Sutlive, T.G., Mitchell, S.D., Maxfield, S.N., McLean, C.L., Neumann, J.C., Swiecki, C.R., ... Flynn, T.W. (2004). Identification of individuals with patellofemoral pain whose symptoms improved after a combined program of foot orthosis use and modified activity: A preliminary investigation. Physical Therapy, 84, 49-61.

Thijs. Y., Van Tiggelen, D., Roosen, P., De Clercq, D., \& Witvrouw, E. (2007). A prospective study on gait-related intrinsic risk factors for patellofemoral pain. Clinical Journal of Sport Medicine, 17(6), 437-445.

Thomee, R., Renstrom, P., Karlsson, J., \& Grimby, G. (1995). Patellofemoral pain syndrome in young women. I - A clinical analysis of alignment, pain parameters, common symptoms and functional activity level. Scandinavian Journal of Medicine \& Science in Sports, 5, 237-244.

Thomee, R., Renstrom, P., Karlsson, J., \& Grimby, G. (1995). Patellofemoral pain syndrome in young women. II - Muscle function in patients and healthy controls. Scandinavian Journal of Medicine \& Science Sports, 5, 245-251. doi: 10.1111/j.1600-0838.1995.tb00041.x

Tiberio, D. (1987). The effect of excessive subtalar joint pronation on patellofemoral mechanics: A theoretical model. The Journal of Orthopaedic and Sports Physical Therapy, 9(4), 160-165. doi: 10.2519/jospt.1987.9.4.160 
Tumia, N., \& Maffuli, N. (2002). Patellofemoral pain in female athletes. Sports Medicine and Arthroscopy Review, 10(1), 69-75.

Venturini, C., Morato, F., Michetti, H., Russo, M., \& Carvalho, V.D. (2006). Estudo da associação entre dor patelofemoral e retropé varo. Acta Fisiátrica, 13(2), 70-73.

Vogelbach, W.D., \& Combs, L.C. (1987). A biomechanical approach to the management of chronic lower extremity spathologies as they relate to excessive pronation. Journal of Athletic Training, 22, 6-16.

Whittle, M. (1993). Gait analysis: An introduction. Oxford: Butterworth- Heinemann.

Witvrouw, E., Lysens, R., Bellemans, J., Cambier, D., \& Vanderstraeten, G. (2000). Intrinsic risk factors for the development of anterior knee pain in an athletic population: A two-year prospective study. The American Journal of Sports Medicine, 28(4), 480-489.

Todo o conteúdo da revista Motricidade está licenciado sob a Creative Commons, exceto quando especificado em contrário e nos conteúdos retirados de outras fontes bibliográficas. 